\title{
A NOTE ON MEANS OF ENTIRE FUNCTIONS
}

\section{T. V. LAKSHMINARASIMHAN}

Let $f(z)$ denote an entire function of order $\rho$ and lower order $\lambda$, $0 \leqq \lambda, \rho \leqq \infty$, and let us define

$$
\begin{aligned}
\sigma_{\delta}(r) & =\left(\frac{1}{2 \pi} \int_{0}^{2 \pi}\left|f\left(r e^{i \theta}\right)\right|^{\delta} d \theta\right)^{1 / \delta}, \quad 0<\delta<\infty, \\
\sigma_{\delta, \alpha}(r) & =r^{--1} \int_{0}^{r} u^{\kappa} \sigma_{\delta}(u) d u, \quad 0<\delta<\infty, \quad-1<\kappa<\infty .
\end{aligned}
$$

For the above two functions, we first prove Theorem 1 below by a method different from that in [4]. Theorem 2 which follows is a simple deduction from Theorem 1 .

We require the following lemmas:

Lemma 1 ([2, Theorem 148]). If $\phi, \psi$ and $\phi^{\prime} / \psi^{\prime}$ are positive increasing functions of $r$ and if $\phi(0)=\psi(0)=0$, then $\phi / \psi$ is an increasing function for $r>0$.

Lemma 2 ([3, Lemma 2]).

$$
\lim _{r \rightarrow \infty} \sup _{\inf } \frac{\log \log \sigma_{\delta, x}(r)}{\log r}={ }_{\lambda}^{\rho}, \quad 0 \leqq \lambda, \quad \rho \leqq \infty .
$$

LEMMA 3. (i) $r^{n+1} \sigma_{\delta}(r)$ is a convex function of $r^{n+1} \sigma_{\delta, k}(r)$;

(ii) $\sigma_{\delta}(r) / \sigma_{\delta, s}(r)$ is an increasing function of $r$.

Proof. Rahman [3, Lemma 3] has proved Lemma 3 (i), with a negligible difference in the definitions of $\sigma_{\delta}(r)$ and $\sigma_{\delta, \alpha}(r)$ and also assuming $k \geqq 0$ instead of $k>-1$. As in his proof, our definitions easily lead to

$$
\frac{d\left\{r^{\kappa+1} \sigma_{\delta}(r)\right\}}{d\left\{r^{\kappa+1} \sigma_{\delta, \kappa}(r)\right\}}=\kappa+1+\frac{d\left\{\log \sigma_{\delta}(r)\right\}}{d\{\log r\}} .
$$

Now, $\log \sigma_{\delta}(r)$ being known to be a convex function of $\log r$ [1], the right-hand member is an increasing function. Hence the left-hand member too is an increasing function, which proves (i) of Lemma 3.

Lastly, by Lemma 1, (i) implies that $r^{k+1} \sigma_{\delta}(r) / r^{k+1} \sigma_{\delta, r}(r)$ is an increasing function, i.e., (i) implies (ii).

Received by the editors January 28, 1963 and, in revised form, November 16, 1964. 
THEOREM 1. For an entire function $f(z)$ of order $\rho$, and lower order $\lambda$, $0 \leqq \lambda, \rho \leqq \infty$,

$$
\lim _{r \rightarrow \infty} \sup \left\{\frac{\sigma_{\delta}(r)}{\sigma_{\delta, \kappa}(r)}\right\}^{1 / \log r}=\frac{e^{p}}{e^{\lambda}}
$$

Proof. It is readily seen from our definitions that

$$
\frac{d}{d r}\left[(\kappa+1) \log r+\log \sigma_{\delta, \kappa}(r)\right]=\frac{1}{r} \frac{\sigma_{\delta}(r)}{\sigma_{\delta, \kappa}(r)}
$$

so that

$$
(\kappa+1) \log \frac{r}{r_{0}}+\log \sigma_{\delta, \kappa}(r)-\log \sigma_{\delta, \kappa}\left(r_{0}\right)=\int_{r_{0}}^{r} \frac{\sigma_{\delta}(u)}{\sigma_{\delta, \kappa}(u)} \cdot \frac{d u}{u},
$$

or

$$
\log \sigma_{\delta, \alpha}(r)=\log \sigma_{\delta, \kappa}\left(r_{0}\right)+\int_{r_{0}}^{r} \frac{m_{\delta, \alpha}(u)}{u} d u
$$

where

$$
m_{\delta, \kappa}(u)=\left[\frac{\sigma_{\delta}(u)}{\sigma_{\delta, \kappa}(u)}-(\kappa+1)\right]
$$

increases as $u$ increases, in virtue of Lemma 3 (ii), and is continuous. Thus for $r>r_{0}$, (2) gives

$$
\log \sigma_{\delta, \alpha}(r)-\log \sigma_{\delta, \alpha}\left(r_{0}\right)<m_{\delta, \alpha}(r)\left[\log r-\log r_{0}\right] .
$$

Using Lemma 2, we get from this

$$
\rho \leqq \limsup _{r \rightarrow \infty} \frac{\log m_{\delta, \alpha}(r)}{\log r}, \quad \lambda \leqq \liminf _{r \rightarrow \infty} \frac{\log m_{\delta, \alpha}(r)}{\log r} .
$$

Again

$$
\log \sigma_{\delta, k}(2 r)-\log \sigma_{\delta, k}\left(r_{0}\right) \geqq \int_{r}^{2 r} \frac{m_{\delta, k}(u)}{u} d u \geqq m_{\delta, k}(r) \log 2,
$$

which gives

$$
\rho \geqq \limsup _{r \rightarrow \infty} \frac{\log m_{\delta, \alpha}(r)}{\log r}, \quad \lambda \geqq \liminf _{r \rightarrow \infty} \frac{\log m_{\delta, \alpha}(r)}{\log r} .
$$

From (4) and (5) we get 
(6)

$$
\lim _{r \rightarrow \infty} \sup \frac{\log m_{\delta, \alpha}(r)}{\log r}={ }_{\lambda}^{\rho} .
$$

The theorem now follows from (3) and (6).

THEOREM 2. For an entire function $f(z)$ of order $\rho$ and lower order $\lambda$, $0 \leqq \lambda, \rho<\infty$,

$$
\begin{gathered}
\log \sigma_{8, k}(r) \sim \log \sigma_{8}(r), \quad r \rightarrow \infty . \\
\text { REFERENCES }
\end{gathered}
$$

1. G. H. Hardy, The mean value of the modulus of an analytic function, Proc. London Math. Soc. (2) 14 (1915), 269-277.

2. G. H. Hardy, J. E. Littlewood and G. Polya, Inequalities, Cambridge Univ. Press, Cambridge, 1952.

3. Q. I. Rahman, On means of entire functions, Quart. J. Math. Oxford Ser. (2) 7 (1956), 192-195.

4. - On means of entire functions. II, Proc. Amer. Math. Soc. 9 (1958), 748750.

Madras Christian College, Madras, India 